\title{
UTILIZAÇÃO DE FIBRAS (EPICARPO) DE BABAÇU COMO MATÉRIA-PRIMA ALTERNATIVA NA PRODUÇÃO DE CHAPAS DE MADEIRA AGLOMERADA ${ }^{1}$
}

\author{
Ademi Moraes Lima ${ }^{2}$, Graziela Baptista Vidaurre ${ }^{3}$, Raimundo de Moraes Lima² e Edvá Oliveira Brito²
}

\begin{abstract}
RESUMO - O objetivo deste trabalho foi avaliar a utilização de fibras (epicarpo) de babaçu (Orbignya spp) em combinação com partículas de Pinus elliottii em diferentes proporções na confecção de chapas de madeira aglomerada, bem como avaliar o efeito de dois teores de adesivo à base de uréia-formaldeído. As chapas foram prensadas a $160^{\circ} \mathrm{C}$, utilizando-se dois níveis de resinas (6 e $8 \%$, base peso seco de partículas) e tempo de prensagem de $10 \mathrm{~min}$, obtendo-se oito tratamentos. Para cada tratamento foram feitas três repetições, totalizando 24 chapas, com densidade nominal de $0,70 \mathrm{~g} / \mathrm{cm}^{3}$. As propriedades avaliadas foram: flexão estática - módulo de ruptura (MOR) e módulo de elasticidade (MOE); tração perpendicular-ligação interna (LI); estabilidade dimensional - inchamento em espessura (IE) 2 e 24 horas e absorção de água (AA) 2 e 24 horas, de acordo com a norma ASTM D 1037-91. O aumento do teor de fibras de babaçu associado ao aumento no teor de adesivo contribuiu para a redução nos valores de inchamento em espessura e absorção de água. O aumento do teor de adesivo de 6 para $8 \%$ foi fundamental para a melhoria das propriedades inchamento em espessura, absorção em água, módulo de ruptura e módulo de elasticidade. Utilizando até $30 \%$ de fibras de babaçu na composição das chapas, os valores de MOR foram superiores aos estabelecidos pela CS 236-66.
\end{abstract}

Palavras-chave: Aglomerados, epicarpo, adesivo e babaçu

\section{USE OF BABACUU STAPLE FIBER AS ALTERNATIVE RAW MATERIAL FOR PANEL PRODUCTION}

\begin{abstract}
The objective of this work was to evaluate the use of babaçu (Orbignya spp) fiber (epicarp) in combination with particles of Pinus elliottii at different ratios in particleboard manufacturing, as well as evaluating the effect of different adhesive contents. The boards were pressed at $160{ }^{\circ} \mathrm{C}$, using two resin levels $-6 \%$ and $8 \%$ (particle dry weight basis) and 10 min pressing time, totaling eight treatments, tree repetitions per treatment, totaling twenty four boards, with $0.70 \mathrm{~g} / \mathrm{cm}^{3}$ nominal density. The evaluated properties were as follows: Static bending - modulus of rupture (MOR) and modulus of elasticity (MOE); Internal bond; Dimensional stability - thickness swelling (IE) 2 and 24 hours and water absorption (AA) 2 and 24 hours, according to ASTM D 1037-91. The increase in babaçu fiber content combined with the increase in resin content contributed to the reduction in thickness swelling and water absorption. The increase in resin content from 6 to $8 \%$ was fundamental to improve thickness swelling properties, water absorption, module of rupture and module of elasticity. Using up to $30 \%$ of babaçu fibers in the panel composition resulted in MOR values above the limit established by CS 236-66.
\end{abstract}

Keywords: particleboards, coconut shell, adhesive and babaçu.

\footnotetext{
${ }^{1}$ Recebido em 20.10.2005 e aceito para publicação em 05.04.2006.

${ }^{2}$ Engenheiros Florestais.

${ }^{3}$ Programa de Pós-Graduação em Ciência Florestal da Universiade Federal deViçosa. E-mail: <grazibaptista@ yahoo.com.br>.
} 


\section{INTRODUÇÃO}

Na floresta de mata seca da Amazônia oriental, principalmente no Maranhão, destacam-se os babaçuais ou cocais de Orbignya spp, que é uma das palmeiras brasileiras mais importantes. A região é considerada a de maior concentração de plantas oleaginosas do mundo e fonte da maior produção extrativista vegetal do país. O gênero Orbignya ocorre em outros países das Américas, do México para o sul (EMBRAPA, 1984), destacando-se os babaçuais da Bolívia.

Segundo Coelho et al. (2002), a zona dos cocais é uma formação de transição entre a floresta amazônica, mata atlântica, caatinga e cerrado, sendo o Estado do Maranhão o maior produtor e responsável por $74 \%$ da produção total. Carvalho (1998) citou que o babaçu é a matéria-prima explorada extrativamente que apresenta enorme potencialidade, tanto do ponto de vista econômico quanto social.

De acordo com Lorenzi (1996), o babaçu é uma palmeira de tronco simples, robusto, imponente, com até $20 \mathrm{~m}$ de altura. Distribui-se isoladamente na floresta ou em áreas abertas, sendo mais freqüente em áreas degradadas, onde a espécie é pioneira dominante. É a maior fonte mundial de óleo silvestre para uso doméstico, tendo utilização industrial em perfumaria, saboaria e lubrificação. O babaçu é considerado o maior recurso oleífero nativo do mundo. É um dos principais produtos extrativistas do Brasil, contribuindo, de maneira significativa, para a economia de alguns estados da federação.

De acordo com a Embrapa (1984), citada por Teixeira e Milanez (2003), os babaçuais se distribuem numa área de aproximadamente 14,5 milhões de hectares, por sete estados brasileiros (Maranhão, Tocantins, Piauí, Goiás, Minas Gerais, Mato Grosso e Espírito Santo), com um potencial produtivo estimado em 15 milhões t/ano, sendo realmente aproveitados somente $30 \%$ do estimado. A coleta e quebra do fruto dessa Palmacea chega a empregar até 2 milhões de pessoas durante o pico da safra. Os principais produtos comerciais extraídos da palmeira de babaçu são o óleo (extraído da amêndoa) e a torta (que resulta do processo). A amêndoa representa $7 \%$ do peso total do fruto e as fibras (epicarpo), $11 \%$, que, se totalmente aproveitadas, gerariam 1,5 milhão de t/ano.

Devido à crescente demanda por matéria-prima pela indústria de base florestal e, conseqüentemente, ao aumento do seu preço, os resíduos de natureza lignocelulósica vêm despertando interesse cada vez maior. Dentre esses resíduos, a casca de babaçu apresenta-se como alternativa, uma vez que há grande disponibilidade desse material em alguns estados do Norte e Nordeste, adicionado ao fato de que ele irá contribuir para amenizar os impactos ambientais. Adicionalmente, deve-se levar em conta que a palmeira de babaçu é uma planta de importância socioeconômica e cultural, pois os povos de sua área de ocorrência utilizam-na para os mais variados fins. Apesar disso, o interesse em estudar tais potencialidades só aconteceu a partir de 1973, com a crise do petróleo, embora de maneira bastante discreta.

Ultimamente, têm-se estudado os resíduos gerados na indústria madeireira, resíduos da atividade agrícola, como bagaço de cana, palha de arroz e de trigo, bem como o mesocarpo de babaçu. Deve-se ressaltar, entretanto, que a maioria dos trabalhos relacionados com essa matéria-prima trata da aplicação energética desses resíduos. Segundo Almeida et al. (2002), os resíduos de babaçu são usados como biomassa para a produção de energia, na maioria das vezes através da queima direta, agregando pouco valor à matériaprima.

O objetivo deste trabalho foi produzir chapas de madeira aglomerada utilizando fibra de babaçu (epicarpo) em combinação com partículas de Pinus elliottii com diferentes níveis de resina.

\section{MATERIAL E MÉTODOS}

A espécie Pinus elliottii foi obtida no campus da Universidade Federal Rural do Rio de Janeiro com 12 anos de idade, onde foram coletadas três (3) árvores, sendo selecionadas aquelas sem defeitos e com fuste retilíneo. As fibras (epicarpo) de babaçu (Orbygnia spp) foram oriundas de Tocantinópolis, TO, provenientes do processo de produção da indústria Tobasa Bioindustrial.

As partículas de Pinus elliottii foram produzidas em um gerador de partículas com comprimento de 3 $\mathrm{cm}$ e espessura de $0,6 \mathrm{~mm}$. Essas partículas passaram por um moinho de martelo com peneira de $12 \mathrm{~mm}$, sendo posteriormente classificadas em peneira de $4,37 \mathrm{~mm}$. As fibras do babaçu foram passadas em um moinho de martelo com peneira de $12 \mathrm{~mm}$, sendo em seguida classificadas em uma peneira de $4,37 \mathrm{~mm}$, para maior homogeneização. 
Na confecção das chapas com as partículas de pinus e fibras de babaçu foram utilizadas as que ficaram retidas na peneira de 4,37 $\mathrm{mm}$. Todas as partículas foram secadas em estufa com ventilação forçada, a $103 \pm 2{ }^{\circ} \mathrm{C}$, até atingir o teor de umidade préestabelecido de $5 \%$. Utilizaramse 6 e $8 \%$ de adesivo uréia-formaldeído, aplicado por meio de aspersão através de uma pistola à base de ar comprimido, em um aplicador do tipo tambor rotatório.

O delineamento experimental foi o inteiramente casualizado em arranjo fatorial $4 \times 2$. Os parâmetros do processo foram: densidade nominal da chapa: $0,70 \mathrm{~g} /$ $\mathrm{cm}^{3}$; conteúdo de adesivo: 6 e $8 \%$; teor de umidade das partículas: $5 \%$; tempo de prensagem: $10 \mathrm{~min}$; tempo de fechamento da prensa: $25 \mathrm{seg}$; e temperatura de prensagem: $160{ }^{\circ} \mathrm{C}$ e $30 \mathrm{~kg} / \mathrm{cm}^{2}$ de pressão específica. Na Tabela 1 , apresentam-se os diferentes tratamentos utilizados.

Tabela 1 - Percentagem de partículas de Pinus e fibras (epicarpo) de babaçu na composição das chapas para cada tratamento

Table 1 -Percentage of Pinus particles and babaçu staple fibers in the composition of panels per treatment

\begin{tabular}{cccc}
\hline Tratamento & $\begin{array}{c}\text { Teor de } \\
\text { adesivo }(\%)\end{array}$ & $\begin{array}{c}\text { Fibra de } \\
\text { babaçu }(\%)\end{array}$ & $\begin{array}{c}\text { Partículas de } \\
\text { pinus }(\%)\end{array}$ \\
\hline T 1 & 6 & 00 & 100 \\
T 2 & 8 & 00 & 100 \\
T 3 & 6 & 10 & 90 \\
T 4 & 8 & 10 & 90 \\
T 5 & 6 & 20 & 80 \\
T 6 & 8 & 20 & 80 \\
T 7 & 6 & 30 & 70 \\
T 8 & 8 & 30 & 70 \\
\hline
\end{tabular}

Após a aplicação do adesivo, as partículas foram colocadas em uma forma quadrada de $40 \mathrm{~cm}$ de lado para moldar o painel e evitar desperdício de partículas. A seguir foi feita uma pré-prensagem a frio $(1 \mathrm{~min})$ para consolidação parcial do colchão. A prensagem foi feita em uma prensa de pratos planos e horizontais com aquecimento elétrico.

Depois de prensadas, as chapas foram climatizadas de acordo com a norma ASTM D 1037-9 até a obtenção do equilíbrio, e os corpos-de-prova foram confeccionados para os ensaios de flexão estática (módulo de ruptura e módulo de elasticidade), inchamento em espessura 2 e 24 h (IE2 e IE24) e absorção de água 2 e 24 h (AA2 e AA24), de acordo com a mesma norma.

Os ensaios mecânicos foram realizados em uma máquina universal de ensaio hidráulica, baseados na norma ASTM D 1037-91. As informações foram obtidas da leitura direta no mostrador analógico da máquina e no caso do ensaio de flexão estática, também em um gráfico carga versus deformação confeccionado para a determinação da carga no limite de elasticidade.

Na Tabela 2, apresentam-se os valores mínimos para as propriedades mecânicas e os valores máximos para o inchamento em espessura.

Para avaliar o efeito dos tratamentos, foi utilizada a análise de variância de todos os ensaios. As análises foram feitas no nível de $95 \%$ de probabilidade, tanto pelo teste de $F$ quanto pelo teste de Tukey (todas as vezes que a hipótese da nulidade foi rejeitada).

Tabela 2 - Valores mínimos exigidos pela Norma Comercial Americana CS 236-66 para as propriedades mecânicas e valores máximos para inchamento em espessura para chapas de partículas

Table 2-Minimum values required by CS 236-66 for mechanical properties and maximum values for swelling thickness for particle panels

\begin{tabular}{|c|c|c|c|c|c|c|}
\hline $\begin{array}{l}\text { Tipo } \\
\text { (uso) }\end{array}$ & $\begin{array}{c}\text { Densidade }\left(\mathrm{g} / \mathrm{cm}^{3}\right) \\
\text { Média mín }\end{array}$ & $\begin{array}{c}\text { Classe }^{3} \\
\text { Média mín }\end{array}$ & $\begin{array}{l}\text { Módulo de } \\
\text { ruptuta } \\
\left(\mathrm{kgf} / \mathrm{cm}^{2}\right) \\
\text { Média mín }\end{array}$ & $\begin{array}{c}\text { Módulo de } \\
\text { elasticidade } \\
\left(\mathrm{kgf} / \mathrm{cm}^{2}\right) \\
\text { Média mín }\end{array}$ & $\begin{array}{c}\text { Ligação } \\
\text { interna } \\
\left(\mathrm{kgf} / \mathrm{cm}^{2}\right) \\
\text { Média mín }\end{array}$ & $\begin{array}{c}\text { Inchamento } \\
\text { em espessura } \\
(\%)\end{array}$ \\
\hline \multirow{6}{*}{$1^{1}$} & A (alta, igual ou & 1 & 168 & 24500 & 14,0 & 55 \\
\hline & superior a $0,80 \mathrm{~g} / \mathrm{cm}^{3}$ ) & 2 & 236 & 24500 & 9,8 & 55 \\
\hline & B (média,entre & 1 & 112 & 24500 & 4,9 & 35 \\
\hline & 0,60 e $\left.0,80 \mathrm{~g} / \mathrm{cm}^{3}\right)$ & 2 & 168 & 28000 & 4,2 & 30 \\
\hline & C (baixa, abaixo & 1 & 56 & 10500 & 1,4 & 30 \\
\hline & de $\left.0,60 \mathrm{~g} / \mathrm{cm}^{3}\right)$ & 2 & 98 & 17500 & 2,1 & 30 \\
\hline \multirow{4}{*}{$2^{1}$} & A (alta, igual ou & 1 & 168 & 24500 & 8,7 & 55 \\
\hline & superior a $0,80 \mathrm{~g} / \mathrm{cm}^{3}$ ) & 2 & 238 & 35000 & 28,0 & 55 \\
\hline & B (média, entre & 1 & 126 & 17500 & 4,55 & 35 \\
\hline & 0,60 e $\left.0,80 \mathrm{~g} / \mathrm{cm}^{3}\right)$ & 2 & 175 & 31500 & 4,2 & 35 \\
\hline
\end{tabular}

${ }^{1}$ Tipo 1 - Chapa de partículas (geralmente feita com resina uréia-formaldeído) adequada para aplicação em interiores.

${ }^{2}$ Tipo 2 - Chapa de partículas feita com resina durável e altamente resistente à umidade e calor (geralmente resina fenólica) adequada para aplicações em interiores e certas aplicações em exteriores quando assim classificadas. ${ }^{3}$ Classe - Classificação de resistência baseada nas propriedades dos painéis produzidos. FONTE: Brito (1995). 


\section{RESULTADOS E DISCUSSÃO}

\subsection{Flexão estática e ligação interna}

Na Tabela 3, apresentam-se os valores médios encontrados nos testes de flexão estática.

Observa-se, na Tabela 3, que o maior módulo de ruptura (MOR) encontrado foi no tratamento $\mathrm{T} 4(8 \%$ de resina e $10 \%$ de fibras de babaçu na mistura com partículas de Pinus), que apresentou um valor médio de $267,6 \mathrm{kgf} / \mathrm{cm}^{2}$, porém não diferenciou estatisticamente dos demais tratamentos. No entanto, pode-se observar que o tratamento que obteve o menor valor de MOR foi o T7 (6\% de resina e 30\% de fibras de babaçu, na mistura com partículas de Pinus). Essas chapas apresentaram um valor médio de $181,8 \mathrm{kgf} / \mathrm{cm}^{2}$. Portanto, pode-se constatar que não houve relação da taxa de fibras com a resistência à flexão estática.

Com relação ao adesivo, à medida que aumentou o teor de adesivo, houve elevação dos valores de MOR em todos os tratamentos. Pode-se observar também que, usando até $30 \%$ de fibras de babaçu na composição das chapas, foram obtidos valores de MOR superiores aos estabelecidos pela norma CS 236-66 e também superiores aos encontrados por Brito e Silva (2002), em chapas de partículas produzidas com duas granulometrias diferentes das de madeira $(2,01-0,61$ mm e 5,68-0,61 mm) a partir das espécies Gmelina arborea, Linn. Roxb. e Samanea saman (Jacq) Merr. com o mesmo tipo de adesivo uréia-formaldeido. $\mathrm{O}$ MOR encontrado por esses autores na espécie Gmelina arborea foi de 111,9 e 140,2 kgf/ $\mathrm{cm}^{2}$, nas granulometrias anteriormente mencionadas, e na Samanea saman MOR encontardo foi de 128,2 e $84,1 \mathrm{kgf} / \mathrm{cm}^{2}$.

Tabela 3 -Flexão estática e ligação interna das chapas produzidas Table 3 - Panel static bending and internal bond

\begin{tabular}{|c|c|c|c|}
\hline \multirow[t]{2}{*}{ Tratamentos } & \multicolumn{3}{|c|}{ Propriedade Avaliada } \\
\hline & $\begin{array}{c}\text { Módulo de } \\
\text { ruptura } \\
(\mathrm{MOR}) \\
\left(\mathrm{kgf} / \mathrm{cm}^{2}\right)\end{array}$ & $\begin{array}{c}\text { Módulo de } \\
\text { elasticidade } \\
(\mathrm{MOE}) \\
\left(\mathrm{kgf} / \mathrm{cm}^{2}\right)\end{array}$ & $\begin{array}{c}\text { Ligação } \\
\text { interna } \\
(\mathrm{LI}) \\
\left(\mathrm{kgf} / \mathrm{cm}^{2}\right)\end{array}$ \\
\hline $\mathrm{T} 1$ & $223,5^{\text {ab }}$ & $24206^{b c}$ & $4,10^{\mathrm{a}}$ \\
\hline $\mathrm{T} 2$ & 237,7 ab & $27791^{c}$ & $4,20^{a}$ \\
\hline T 3 & 208,3 ab & 22147 abc & 4,32 a \\
\hline $\mathrm{T} 4$ & 267,6 b & 26539 bc & 4,62 a \\
\hline T 5 & 227,2 ab & 23584 bc & 4,30 a \\
\hline T 6 & 247,5 b & 28435 & 4,09 a \\
\hline T 7 & 181,8 ab & 20339 ab & 3,93 a \\
\hline T 8 & $204,3^{\text {ab }}$ & 22749 bc & $3,84^{a}$ \\
\hline
\end{tabular}

Letras iguais na mesma coluna não diferem estatisticamente, a 95\% de probabilidade.
Em relação ao módulo de elasticidade, o melhor tratamento foi T6 (8\% de adesivo e $20 \%$ de fibras de babaçu na mistura com partículas de Pinus), que apresentou um valor médio de $28.435 \mathrm{kgf} / \mathrm{cm}^{2}$, diferenciando-se estatisticamente do tratamento $\mathrm{T} 7$ (6\% de resina e $30 \%$ de fibras de babaçu na mistura com partículas de Pinus), que apresentou o menor valor médio $\left(20.339 \mathrm{kgf} / \mathrm{cm}^{2}\right)$. Os tratamentos T1, T2, T4, e T6 exibiram valores superiores aos encontrados por Brito e Silva (2002), ao estudarem as propriedades de chapas de partículas de Gmelina arborea Linn. Roxb. (23080 e $23850 \mathrm{kgf} / \mathrm{cm}^{2}$ - para granulometria das partículasde $2,01-0,61 \mathrm{~mm}$ e $5,68-0,61 \mathrm{~mm}$, respectivamente) e Samanea samaen (Jacq) Merr (22.419, 15.910 e $20.339 \mathrm{kgf} / \mathrm{cm}^{2}$ ), utilizando-se $10 \%$ do mesmo adesivo uréia-formaldeído. Apesar de o melhor tratamento ter sido o T6 (20\% de fibras), não foi constatada melhoria de MOE com o aumento do porcentual de fibras de babaçu na composição das chapas. No entanto, com o aumento do teor de adesivo, constatou-se aumento dos valores do MOE.

Para a ligação interna (LI), a análise de chapas produzidas com partículas apenas de Pinus (T1 e T2) e com a mistura de $10 \%$ de fibras de babaçu misturadas com Pinus (T3 e T4) demonstrou um aumento na LI quando se aumentou o teor de adesivo, apesar de não ter sido significativo. Essa melhoria em função do aumento do teor de adesivo também foi constatado por Nunes et al. (1999), ao estudarem a influência de vários teores do mesmo adesivo na produção de chapas de partículas do estipe de Euterpe edulis Martius. O tratamento que apresentou o menor valor médio para LI foi T8, com $3,84 \mathrm{kgf} / \mathrm{cm}^{2}$. Os valores médios para LI em todos os tratamentos mostraram-se inferiores aos encontrados por Brito e Silva (2002), estudando as propriedades de chapas de partículas de Gmelina arborea Linn. Roxb. (6,0 e $6,53 \mathrm{kgf} / \mathrm{cm}^{2}-$ para granulometria das partículas de 2,01-0,61 $\mathrm{mm}$ e 5,68 -0,61 $\mathrm{mm}$, respectivamente) e Samanea saman (Jacq) Merr. (7,18 e $7,62 \mathrm{kgf} / \mathrm{cm}^{2}$ ) a $10 \%$ de adesivo uréia-formaldeido, bem como inferiores aos valores mínimos da norma CS 236-66.

\subsection{Inchamento em espessura e absorção de água}

Na Tabela 4 são apresentados os valores médios encontrados para o inchamento em espessura (IE) e absorção de água (AA) 2 e 24 h, nos tratamentos realizados. 
Tabela 4 - Valores médios de inchamento em espessura (IE) e absorção em água (AA) por 2 e 24 horas Table 4 -Average values of thickness swelling and water absorption for 2 and 24 hours

\begin{tabular}{|c|c|c|c|c|}
\hline \multirow[t]{2}{*}{ Tratamentos } & \multicolumn{4}{|c|}{ Propriedade Avaliada } \\
\hline & IE $2 \mathrm{~h}(\%)$ & IE $24 \mathrm{~h}(\%)$ & AA $2 \mathrm{~h}(\%)$ & AA $24 \mathrm{~h}(\%)$ \\
\hline $\mathrm{T} 1$ & 49,70969 b & 57,59 c & $111,98^{b}$ & 125,85 b \\
\hline $\mathrm{T} 2$ & 38,28 ab & $43,46^{a b c}$ & $87,33^{a b}$ & $98,23^{a b}$ \\
\hline T 3 & 37,51 ab & 43,33 abc & $81,46^{a}$ & 91,93 a \\
\hline $\mathrm{T} 4$ & 33,90 ab & $39,60^{\mathrm{ab}}$ & 77,42 a & 86,58 \\
\hline T 5 & $45,32 \mathrm{ab}$ & 53,40 abc & $91,00^{a b}$ & 102,47 ab \\
\hline T 6 & $40,53 \mathrm{ab}$ & $46,24^{a b c}$ & $88,78^{\text {ab }}$ & $100,05^{a b}$ \\
\hline $\mathrm{T} 7$ & 39,96 ab & $47,54^{a b c}$ & 94,48 ab & 105,93 ab \\
\hline $\mathrm{T} 8$ & 32,10 a & 37,86 a & 80,82 a & $90,04^{\mathrm{a}}$ \\
\hline
\end{tabular}

Letras iguais na mesma linha não diferem estatisticamente no nível de $95 \%$ de probabilidade

O inchamento em espessura (IE) é uma alteração sofrida pelas chapas de partículas quando expostas à umidade, o que torna um fator limitante em relação ao uso do produto (BRITO e SILVA, 2002). A análise de variância indicou que, para o inchamento em espessura (IE) por 2 e $24 \mathrm{~h}$, o melhor tratamento foi o T8 (8\% de adesivo e $30 \%$ de fibras de babaçu na mistura com partículas de Pinus), que apresentou valores médios de 32,10 e $37,86 \%$, respectivamente. Observou-se que os valores médios encontrados de IE ficaram ligeiramente inferiores aos estabelecidos na norma (Tabela 2). Cabe mencionar que o T8 só diferenciou estatisticamente do tratamento T1 (chapas confeccionadas com $6 \%$ de adesivo e $100 \%$ de partículas de Pinus), que apresentou valor médio de IE 49,70 e 57,59\%, respectivamente. Em relação ao adesivo houve melhoria do IE à medida que se aumentou o nível do adesivo, constatado também por Nunes, et al. (1999), embora a diferença não tenha sido significativa.

Para a absorção de água (AA) tratamento T4 (8\% de adesivo, $10 \%$ de fibras de babaçu na mistura com partículas de Pinus), apresentou os maiores valores médios de AA $(77,42$ e $86,58 \%)$ para AA 2 h e AA 24 $\mathrm{h}$, respectivamente, só havendo diferença estatística com o tratamento $\mathrm{T} 1$ - chapas confeccionadas com $6 \%$ de adesivo e $100 \%$ de partículas de Pinus.

\section{CONCLUSÕES}

- A utilização de fibras de babaçu, mesmo em seu nível mais alto (30\%), proporcionou resultados iguais, estatisticamente, àqueles encontrados em chapas fabricadas com partículas de Pinus para as propriedades mecânicas.
- O aumento no teor de fibras de babaçu associado ao incremento no teor de adesivo, contribuiu para a redução nos valores de inchamento em espessura (IE) e absorção de água (AA).

- A resistência à tração das chapas (ligação interna) não foi influenciada pelo aumento no teor de adesivo, nem pelo aumento do porcentual de fibras de babaçu sobre as partículas de Pinus.

- O aumento do teor de adesivo de 6 para $8 \%$ foi fundamental para a melhoria das propriedades inchamento em espessura (IE), absorção em água (AA), módulo de ruptura (MOR) e módulo de elasticidade (MOE).

- Utilizando até $30 \%$ de fibras de babaçu na composição das chapas, os valores de MOR foram superiores aos estabelecidos pela Norma Comercial Americana CS 236-66.

\section{AGRADECIMENTOS}

À empresa Borden Química, pelo fornecimento do adesivo à base de uréia-formaldeído (Cascamite PB 2346); e à Tobasa Bioindustrial, pelo fornecimento do epicarpo de babaçu.

\section{REFERÊNCIAS BIBLIOGRÁFICAS}

ALMEIDA, R. R.; Del MENEZZI, C. H. S.; TEIXEIRA, D. E. Utilization of the coconut shell of babaçu (Orbignya sp.) to produce cementbonded particleboard, Bioresource

Technology, v. 85, n. 2, p.159-163, 2002.

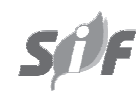

R. Árvore, Viçosa-MG, v.30, n.4, p.645-650, 2006 
AMERICAN SOCIETY FOR TESTING

MATERIALS - ASTM. Standard methods of evaluating the properties of wood base fiber and particle materials. ASTM D - 1037-91 Philladelphia: 1987. v. 04.09 (Annual Book of ASTM standards).

BRITO, E. O.; SILVA,G. C.Propriedades de chapas de partículas produzidas a partir de Gmelina arbórea Linn Roxb. e Samanea saman (Jacq.) Merr. Floresta e Ambiente, v. 9, n. 1, p.127-134, 2002.

BRITO, E.O. Produção de chapas de partículas de madeira a partir de maravalhas de Pinus elliotti plantado no Sul do Brasil. 1995. 127f. Tese (Doutorado em Engenharia Florestal), Universidade Federal do Paraná, Curitiba, 1995.

BRITO, E. O. A viabilidade de utilização de espécies de Pinus para a produção de chapas de composição estruturais “waferboards". 1984. 104f. Dissertação (Mestrado em Engenharia Florestal). Universidade Federal do Paraná, Curitiba, 1984.

CARvalho, J. H. Programa Nacional de Pesquisa de Babaçu: Uma experiência a ser continuada e aplicada. Teresina: Embrapa Meio Norte, 1998. 4p. (EMBRAPA Doc./32).

COElho, S. T. et al. Panorama do Potencial de Biomassa no Brasil. Brasília: ANEE, 2002. 80p.

R. Árvore, Viçosa-MG, v.30, n.4, p.645-650, 2006
EMPRESA BRASILEIRA DE PESQUISA

AGROPECUÁRIA - EMBRAPA. Departamento de Difusão de Tecnologia. Babaçu, Programa Nacional de Pesquisa. Brasília: 1984. 89 p.

LIMA, A. M.;LIMA, R. M.; BRITO, E. O.

Produção de aglomerados a partir de partículas "strand" combinadas com maravalhas. Ciências da Vida, v. 22, n. 2, p. 175-179, 2002.

LOREnZI, H. et al. Palmeiras no Brasil: exóticas e nativas. Nova Odessa: Plantarum, 1996. 303 p.

MENDES, M. L. Pinus spp. Na produção de painéis de partículas orientadas (OSB). 2001. 156f. Tese (Doutorado em Ciências Florestais) - Universidade Federal do Paraná, Curitiba, 2001.

NORMEN FüR HOLZFASERPLATEN SPANPLATTEN SPERHOLZ. Testing of wood chipboards, bending test, determination of bending strength, DIN 52362. Germany: 1982. p. 39-40

NUNES, W. H.; ANDRADE, A. M.; BRITO, E. O. Produção de chapas de partículas do estipe de Euterpe edulis Martius (Palmiteiro). Floresta e Ambiente, v. 6, n. 1, p. 95-105, 1999.

TEIXEIRA, M. A.; MILANEZ, L. F. Caracterização energética do babaçu e análise do potencial de cogeração. 2003. 198f. Tese (Doutorado em Engenharia Mecânica) - Universidade Estadual de Campinas, Campinas, 2003. 